Cinémas

Revue d'études cinématographiques

Journal of Film Studies

\title{
Il faut trouver la voix
}

\section{Jean Châteauvert}

Volume 3, numéro 1, automne 1992

Cinéma et Musicalité

URI : https://id.erudit.org/iderudit/1001180ar

DOI : https://doi.org/10.7202/1001180ar

Aller au sommaire du numéro

Éditeur(s)

Cinémas

\section{ISSN}

1181-6945 (imprimé)

1705-6500 (numérique)

Découvrir la revue

Citer cet article

Châteauvert, J. (1992). Il faut trouver la voix. Cinémas, 3(1), 64-77. https://doi.org/10.7202/1001180ar

\section{Résumé de l'article}

L'article interroge les paramètres qui déterminent le rôle el les fonctions attribués à un personnage suivant les caractéristiques de sa voix. En premier lieu, on y pointe les caractéristiques physiques et énonciatives qui modulent la perception d'une voix. Dans un second temps, on interroge l'impact de deux de ces paramètres, le grain et le timbre, sur la perception spectatorielle suivant que le personnage est visualisé ou non. L'analyse fait ressortir que certaines caractéristiques positionnent le personnage comme objet (et non pas comme sujet) et peuvent même invalider la portée effective (perlocutoire) du discours verbal. d'utilisation que vous pouvez consulter en ligne.

https://apropos.erudit.org/fr/usagers/politique-dutilisation/ 


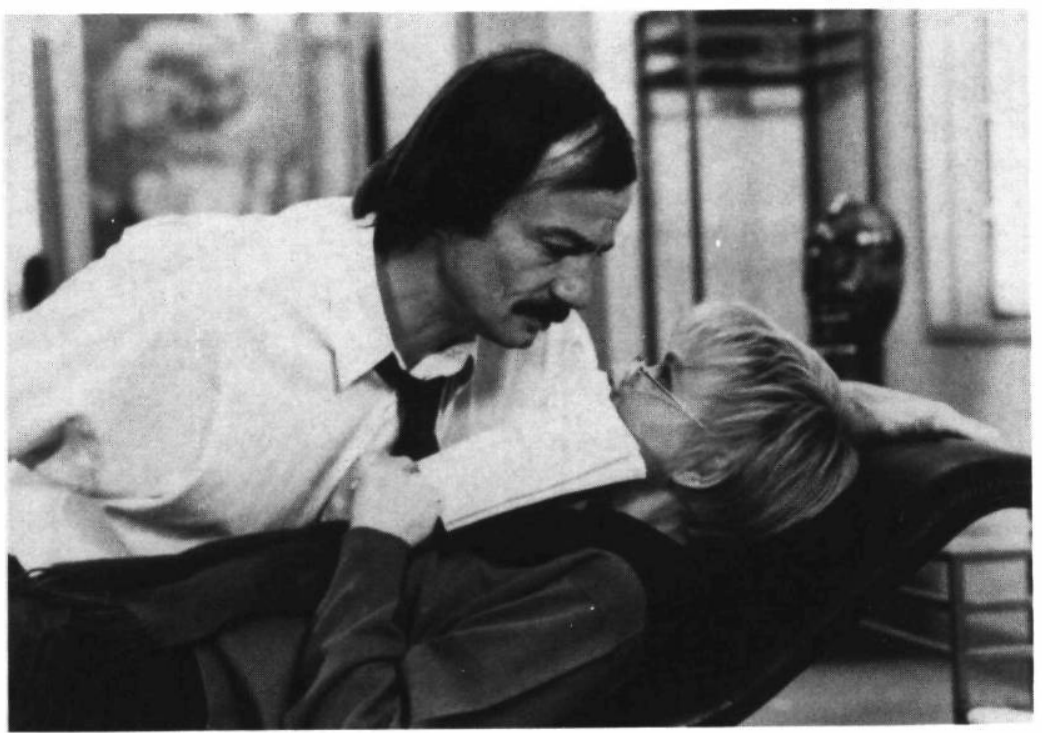

La Lectrice de Michel Deville (1988)

Coll. Cinémathèque québécoise 


\title{
Il faut trouver la voix
}

\section{Jean Châteauvert}

\begin{abstract}
RÉSUMÉ
L'article interroge les paramètres qui déterminent le rôle et les fonctions attribués à un personnage suivant les caractéristiques de sa voix. En premier lieu, on y pointe les caractéristiques physiques et énonciatives qui modulent la perception d'une voix. Dans un second temps, on interroge l'impact de deux de ces paramètres, le grain et le timbre, sur la perception spectatorielle suivant que le personnage est visualisé ou non. L'analyse fait ressortir que certaines caractéristiques positionnent le personnage comme objet (et non pas comme sujet) et peuvent même invalider la portée effective (perlocutoire) du discours verbal.
\end{abstract}

\begin{abstract}
This article studies the parameters of the voice that determine the position and functions of a character. One shows the physical and enunciative components that influence the perception of a voice. In a second time, the author studies the influence of two parameters, the tone and the specific sound of a voice (grain de la voix) on the spectator's perception following that the character is visualized or not. The analysis points out that some characteristics determine the position of the character as an object (by opposition with a subject) and generate a special effect of his discourse (perlocutionnary effect).
\end{abstract}

On peut facilement avoir l'impression que la sonorité d'un film, dans le sens de ses qualités sonores, se limite à sa trame musicale et aux effets sonores qui l'accompagnent. Or, dès que l'on écoute un film d'un peu plus près, dès lors que l'on porte attention aux jeux de sonorité mis en scène dans une bande-son, il apparaît 
rapidement que le son d'un film repose sur ses qualités musicales et ses effets sonores mais aussi sur les voix de ses acteurs qui induisent une tonalité et une texture dans le film. D'aucuns seraient sans doute tentés d'y voir là un élément accessoire, marginal dans la texture sonore d'un film. Pourtant, il suffit de se fermer les yeux pour soudainement entendre la richesse et la saveur de la voix des acteurs, pour voir surgir le grain et les caractéristiques spécifiques de leur voix. C'est cette question de la voix, de ses caractéristiques et qualités spécifiques qu'on abordera ici : on va tenter d'identifier les paramètres qui conditionnent le choix d'une voix, c'est-à-dire les caractéristiques qu'on demande à une voix pour remplir telle ou telle fonction au sein d'un film.

Dans cette exploration du casting vocal, on essaiera de signaler les différents paramètres avec lesquels on juge une voix mais aussi des problématiques telles que le rôle de la femme dans l'économie filmique, l'influence de la voix sur notre perception du ou des personnages et, enfin, le rapport entre la voix et la visualisation du personnage. En bref, il s'agira de déterminer les caractéristiques qui déterminent la spécificité d'une voix et de signaler leurs effets sur notre perception du personnage.

\section{Les caractéristiques physiques de la voix}

Dès lors qu'une voix se fait entendre, et avant même d'en écouter le discours, celle-ci nous livre une grande quantité d'informations qu'on peut, pour les besoins de l'analyse, regrouper sous six rubriques.

Une voix a un certain volume : elle peut être criée, chuchotée, déclamée, c'est-à-dire adopter diverses intensités depuis les voix très fortes, comme celle qui ponctue «News on the March» dans Citizen Kane (Welles, 1941), d'une remarquable ampleur, jusqu'à ces voix chuchotées comme celle du personnage incarné par Mia Farrow, voix qui filtre par la trappe d'aération dans Another Woman (Une autre femme, Allen, 1988).

Une seconde caractéristique, plus contingente, est celle du rythme : le rythme de la voix peut varier depuis l'extrême lenteur, comme les voix qui accompagnent India Song (Duras, 1975), jusqu'à un débit très rapide, comme celui de l'amie qui lit une nouvelle dans le film La Lectrice (Deville, 1988). Si la dimension du rythme passe souvent inaperçue, elle n'en demeure pas moins une caractéristique qui a une importance considérable, notamment par son influence sur notre émotivité. Dans la culture occidentale, le rythme est mis en corrélation avec des émotions bien distinctes : ainsi on rattache volontiers à un lento un sentiment de tristesse, tandis qu'un allegro con vivace sera davantage perçu comme plus dynamique, plus joyeux. Aussi l'adoption d'un rythme implique, 
d'une façon générale, une tonalité affective, en corrélation avec un registre de discours.

Le plan sonore, ou spatialisation, permet de distinguer la présence quasi ontologique de la voix telle qu'elle se pratique à la radio, de la voix spatialisée, située dans un lieu. Pour ne donner qu'un exemple, la voix de Truffaut qui explique ce qu'est un réalisateur dans La Nuit américaine (1973) jouit d'une présence immédiate qui élimine tout repère spatial tandis que, dans le même film, l'écho lié à la voix du régisseur qui dirige les figurants à l'aide d'un porte-voix situe d'emblée la voix dans un vaste lieu. Dans le cinéma narratif classique, une voix spatialisée est en général soumise à la diégèse visualisée tandis qu'on décontextualise volontiers la voix qui participe des niveaux enchâssants telle la voix du narrateur (Chion, 1982 : p.58).

La durée correspond au temps de présence de la voix dans le discours filmique : on note des variations depuis la saturation totale de l'espace sonore, la voix de Guitry narrant tout le récit enchâssé dans Le Roman d'un tricheur (1936), jusqu'à la présence on ne peut plus discrète de la voix du «narrateur» dans Ma nuit chez Maud (Rohmer, 1969) qui se réduit à deux répliques, l'une au début et l'autre à la fin du film.

À travers la voix, on entend aussi le personnage qui s'exprime, le personnage comme entité physique qui a ses caractéristiques propres. La voix :

(...) fait entendre un corps qui, certes n'a pas d'état civil, de «personnalité», mais qui est tout de même un corps séparé (...). Le grain de la voix ce serait cela : la matérialité du corps parlant sa langue maternelle (Barthes, p. 238).

Le grain de la voix est une véritable mine d'informations sur l'individu en ce qu'il témoigne :

De l'être du sujet : sexe, âge, identité: origine socio-culturelle et empreinte vocale particulière (voice print); typage (façon de poser la voix, accents, habitudes articulatoires) mais aussi timbre de voix personnelle (Bailblé, 1979 : p. 53).

Ainsi, à une voix tonitruante on rattachera volontiers un corps massif, à une voix timorée, un corps plutôt chétif, à une voix cassante, une gestuelle brusque, à une voix caressante, une certaine souplesse et ainsi de suite. Pour ne citer que deux exemples, dans le film Kiss Me Deadly (En quatrième vitesse, Aldrich, 1955), le chef des bandits qui injecte un sérum de vérité au détective Mike Hamer est toujours présenté de façon tronquée, on ne voit jamais son visage, mais le seul grain de sa voix suffit pour dire qu'il s'agit d'un homme mature, posé, rationnel, qui semble avoir une certaine 
éducation. Pareillement, dans le film The Name of the Rose, (Le Nom de la rose, Annaud, 1985), le portrait du narrateur repose sur le seul grain de la voix : c'est en s'appuyant sur la sonorité de la voix qu'on se construit une image d'un homme d'un grand âge, qui a atteint une certaine sérénité.

Enfin, le dernier paramètre qu'on retiendra ici est celui du timbre : le timbre de la voix permet l'identification de certaines voix, mais aussi ajoute des connotations particulières tant au discours qu'à son locuteur. Ainsi la voix de «la grande actrice» que double l'héroïne dans Singin'in the Rain (Chantons sous la pluie, Donen-Kelly, 1952) suffit à discréditer le personnage par son seul timbre : le personnage féminin apparaît ridicule par son timbre trop clair, par sa voix trop aiguë. Â l'opposé, le timbre de voix grave, posé et mature du narrateur Maupassant dans $L e$ Plaisir (Ophüls, 1952) induit une crédibilité et une légitimité incontestables.

Ce sont ces deux derniers paramètres, le grain et le timbre, qui déterminent l'identité d'une voix et permettent de distinguer différents locuteurs par leur seule voix. Ce sont donc à la fois le grain et le timbre qui nous serviront de balises pour notre analyse du casting vocal, en essayant de démarquer l'apport spécifique de l'un et de l'autre.

$\mathrm{Au}$ sein du matériau filmique, on distingue deux contextes d'occurrence qui influent sur notre perception de la voix : le personnage locuteur peut être visualisé — un personnage dialogue avec un tiers - , ou être réduit à sa seule voix, tel le narrateur en voix over qui ponctue quantité de films. Suivant le contexte d'occurrence, les caractéristiques sonores n'ont évidemment pas le même impact, aussi distinguera-t-on dans notre analyse les contextes où le personnage est visualisé des contextes où la voix est acousmatique, c'est-à-dire ces cas où la source n'est pas visualisée.

\section{Le personnage visualisé}

La visualisation du personnage l'expose d'entrée de jeu à ce que Pascal Bonitzer a très justement appelé «la critique du regard» (Bonitzer, p.25), c'est-à-dire que le discours du personnage subit le poids de son contexte d'occurrence : le spectateur peut alors évaluer le dire à l'aune de ce qu'il voit, il peut le confronter avec le corps du locuteur et l'environnement que présente l'image. Dans ce contexte, le grain et le timbre de la voix sont dans un premier temps évalués en regard du portrait physique du personnage : de façon plus ou moins consciente, on compare le corps qui s'entend dans la voix avec le corps auquel on attribue cette voix ${ }^{1}$. S'il peut y avoir des contrastes évidents, comme lorsqu'on attribue une voix de femme à un homme ou lorsqu'on 
confère à un homme fort, viril et musclé une voix haute pour ne pas dire délicate, il demeure que, d'une façon générale, il y a adéquation entre le physique du locuteur et le corps qui se laisse entendre dans la voix.

À ce portrait physique de l'individu, le timbre ajoute une seconde dimension qui influe directement sur notre perception des personnages. Dans Gone With the Wind (Autant en emporte le vent, Fleming, 1939), on ne remarque qu'incidemment la voix de Clark Gable au timbre grave et, moins encore, celle de Vivien Leigh, qui sont pourtant les personnages principaux de cette grande et belle histoire. Les voix que l'on remarque par leur timbre sont celles de la bonne Mammy et de la jeune servante Prissy, respectivement interprétées par les comédiennes noires Hattie McDaniel et Butterfly McQueen : la première se démarque très nettement par son timbre de voix rauque, guttural, son ton cassant et rustre tandis que la seconde se distingue par la hauteur exagérée de sa voix, son débit infantile et son aspect criard. Dans le contexte du film, on remarque que le timbre de l'une et l'autre n'est pas sans correspondance avec leur position au regard du personnage Scarlett O'Hara : Mammy se présente comme une figure d'autorité à l'égard de Scarlett, tel un délégué de la figure parentale tandis que Prissy, infantilisée à la fois dans ses caractéristiques physiques et dans son comportement, se présente comme une enfant soumise à l'autorité de la même Scarlett.

Dans le contexte du film Gone With the Wind, cette caractérisation de ces deux personnages par leur timbre de voix a une seconde incidence : non seulement y discerne-t-on la position des deux personnages à l'égard de l'héroïne mais, qui plus est, elle discrédite les deux personnages et leur discours. Lorsque Prissy ou Mammy parlent, on porte une attention différente à leur discours: on est moins attentif au dit qu'au dire parce que l'une et l'autre ont un timbre de voix marqué. Par comparaison avec les autres personnages, ces deux voix marquées induisent un aspect non sérieux : on ne croit pas à leur discours comme à celui des autres personnages, on ne leur accorde pas la même crédibilité.

Dans le film Amadeus (Forman, 1984), le personnage Mozart se présente comme un adolescent parce que d'une part on lui donne un comportement juvénile - on pense à la première rencontre Mozart-Salieri où Mozart est présenté tandis qu'il joue à cachecache sous les tables avec une soubrette - mais aussi parce qu'on lui attribue un timbre de voix aigu, d'une clarté infantile, en particulier dans ses éclats de rire. Les fervents admirateurs du grand maître se sont d'ailleurs manifestés à la sortie du film pour protester contre le portrait que l'on donnait du génie, certains alléguant «qu'un génie qui compose une telle musique ne pouvait 
avoir cette voix stupide». La caractérisation du timbre de voix de Mozart a pour effet de jeter un discrédit sur le personnage et son discours. Au sein du film cependant, le génie musical de Mozart en sort pour ainsi dire accentué : le maître est d'autant plus génial qu'il était en fait sot, comme si toute son intelligence se résumait à ses talents de compositeur.

On voit paraître à travers ces quelques exemples que le timbre de voix influe directement sur notre perception de la crédibilité d'un personnage visualisé et de son discours. On essaiera d'explorer plus avant les mécanismes sémiotiques mis en jeu par le timbre de la voix en prenant cette fois des exemples de voix acousmatiques, soit des voix over qui, espérons-le, devraient nous permettre de signaler plus spécifiquement l'influence du timbre sur notre perception du discours et du locuteur.

\section{Le personnage non visualisé}

Lorsqu'on parle du discours d'un personnage non visualisé, on peut parler en fait de trois configurations bien distinctes ${ }^{2}$. La première recoupe les exemples analysés plus haut : il s'agit du discours d'un personnage qui amorce un flash back et qui devient, le temps de ce flash back, un discours en voix over. La voix conserve alors les caractéristiques qu'elle avait au moment où le personnage était visualisé; ce que modifie le passage d'une voix in à une voix dite over ce seront, d'une façon générale, tous les indices contextuels tels la spatialisation, les bruits d'ambiance, la présence de l'allocutaire s'il y en a un et d'autres éléments qui renvoient au contexte de locution. Pour ne citer qu'un exemple, dans le film La Lectrice (Deville, 1988), le passage de la scène où le personnage incarné par Miou Miou lit le livre à voix haute au segment correspondant aux péripéties du personnage «la lectrice» va de pair avec une présence immédiate de la voix de la narratrice et une éviction progressive des indices contextuels; ni le grain ni le timbre ne sont affectés par ce passage de la voix in à une voix over, seule change la contextualisation, soit l'ambiance sonore qui s'éclipse discrètement ${ }^{3}$.

La seconde configuration correspond à un type de narration ou de commentaire homodiégétique où le narrateur, en outre d'avoir participé au monde qu'il raconte, soit pour en avoir été le témoin, soit pour y avoir été un des principaux intervenants, demeure de but en blanc dans un registre over au regard de l'image, c'est-àdire qu'à aucun moment on ne voit le personnage faisant son récit et/ou son commentaire, mais il manifeste le même grain de voix que le personnage qu'il était. C'est le cas notamment des narrateurs dans les Contes Moraux d'Eric Rohmer ${ }^{4}$ : dans ces films, le personnage visualisé et le narrateur homodiégétique ont le 
même grain et on confère ainsi, via la reconnaissance de la voix, les attributs du personnage visualisé passé au personnage devenu narrateur dans le présent, le décalage entre les deux étant présumé mince, voire de peu d'importance.

La configuration qui retiendra davantage notre attention correspond aux narrateurs hétérodiégétiques qui racontent une histoire à laquelle ils ne participent en aucune façon, tel celui de To Be Or Not To Be (Jeux dangereux, Lubitsch, 1942), mais aussi aux narrateurs homodiégétiques qui, en raison d'un décalage temporel important, ont un timbre de voix différent de celui du personnage qu'ils étaient. On pense en particulier à ces narrations professées par des adultes qui racontent leurs lointains souvenirs à l'exemple du narrateur qui remémore sa petite enfance dans Les Mistons (Truffaut, 1957) ou à ces récits de personnes très âgées qui rappellent les aventures de leur jeunesse tel le vieillard qui raconte ses tumultueux débuts de vie monastique dans The Name of the Rose. Tant dans le cas de la narration hétérodiégétique que dans celui de la narration homodiégétique marquée par un grain de voix différent pour le personnage visualisé et pour le narrateur, ce sont les seules caractéristiques repérables dans la texture de la voix qui nous permettent de nous construire une image du locuteur. Ainsi, le grand âge du moine narrateur dans The Name of the Rose ne s'établit-il qu'en fonction du grain de la voix over; pareillement l'homme mature qui rappelle son enfance dans Les Mistons est une construction que l'on se forge à partir des seuls indices du grain de sa voix; $a$ fortiori, l'identité du narrateur hétérodiégétique non visualisé, tel celui de To Be Or Not To Be ne repose-t-elle que sur les caractéristiques repérables au sein du discours, mais plus encore dans le portrait que dessine le grain de la voix.

À l'égard de ces voix de personnages non visualisés, on a souvent souligné une certaine standardisation, une uniformisation : lorsqu'une voix over se superpose à l'image, il s'agit généralement d'une voix d'homme dont le grain et le timbre traduisent une maturité et une crédibilité certaines, une voix qui adopte un ton posé qui marque l'assurance et la légitimité de son locuteur. C'est au prix de cette combinaison d'attributs que s'établirait la légitimité et l'autorité du narrateur en voix over. Dans cette «uniformisation de la production", on voit poindre pour notre analyse deux dimensions fondamentales : il semble dans un premier temps que la voix masculine soit privilégiée au regard de la voix féminine; on remarque aussi que le timbre et le grain de la voix semblent influer directement sur la crédibilité du locuteur. On essaiera maintenant d'analyser les conséquences de ces deux dimensions sur le casting d'une voix. 


\section{- La place de la femme au cinéma}

Traditionnellement, la femme a été maintenue dans certains rôles, certaines fonctions à l'intérieur de l'économie narrative. $\mathrm{Ce}$ sexisme est particulièrement fort au niveau de la narration et du commentaire over, traditionnellement réservés aux hommes. Pascal Bonitzer (p. 32) lie cette absence de la voix féminine à l'idéologie dominante tandis que Mary Ann Doane (1980, p. 49) explique cette exclusion par des motifs psychanalytiques, la voix d'autorité étant celle du père, celle de la mère étant reléguée à une fonction réconfortante sans véritable autorité. Sarah Kozloff (pp. 99-100) rejette ces hypothèses et souligne en premier lieu que nombre de documentaires, en particulier les actualités de guerre dans les années 40 , sont narrés par des femmes et qu'on retrouve des films qui présentent un narrateur féminin déclinant un discours à la première personne. En fait, la femme s'exprime mais demeure limitée à des récits à la première personne dans le cinéma de fiction à l'instar de Rebecca (Hitchcock, 1940), A Letter to Three Wives (Chaînes conjugales, Mankiewicz, 1949) ou Reversal of Fortune (Schrœder, 1990).

Suivant Kozloff, les causes de cette restriction seraient multiples: d'une part, la tradition orale du conte narré par le ménestrel influerait sur notre perception du grand imagier et de ses délégués, en l'occurrence le narrateur verbal, et induirait l'idée qu'il s'agit d'un homme; d'autre part, le rôle classique de la femme comme objet de désir plutôt que comme sujet se conjuguerait au niveau du discours verbal par une exclusion de la position énonciative sinon sous forme d'une présence contextualisée, c'est-à-dire comme objet au sein d'un discours enchâssant. Enfin, la tradition littéraire aurait circonscrit la narration des femmes à des romans autobiographiques ou homodiégétiques (pp. 99-100). Aussi, la restriction de la femme serait somme toute relative : la véritable forclusion toucherait surtout les récits hétérodiégétiques. On notera cependant des contre-exemples notoires qui mettent en scène des narrateurs hétérodiégétiques féminins tels $L a$ Salamandre (Tanner, 1972) et Mon oncle d'Amérique (Resnais, 1980), sans que soit, de quelque façon, remis en cause l'autorité et la légitimité de la narratrice. C'est dire que l'usage n'a rien d'incontournable.

\section{- Le timbre, le grain et la tonalité de la voix Pascal Bonitzer a souligné:}

C'est cela que la voix off, en tant que commentaire, ne doit ni ne peut être: une voix brûlante - la voix éphémère, fragile, malaisée des révoltés (p.27). 
En d'autres termes, une voix qui laisse entendre sa matérialité, qui laisse trop paraitre son grain, sa texture, influe directement sur notre perception du discours : la voix trop marquée «désamorce le savoir qu'impose cette voix» (Bonitzer, pp.29-31) et peut affecter le sens du discours. La thèse se vérifie partiellement dans le discours des personnages visualisés qui amorcent des flash backs dans le film Fellini Satyricon (Fellini, 1969) où les discours sont marqués par leur timbre de voix; ainsi la voix du vieil homme qui raconte l'histoire terrible des amours d'EEnothea apparaît extrêmement perturbante pour le spectateur précisément parce que le timbre de voix du personnage, trop aigu et presque criard, se laisse entendre pour tel; la voix marquée par son timbre vient en quelque sorte mettre en doute la légitimité du personnage et de son discours.

Les exemples de voix over hétérodiégétiques marquées, dont le personnage source n'est jamais visualisé, sont en fait rarissimes tant et si bien qu'on ne retrouve guère que des cas particuliers tel le discours du commentateur de Las Hurdes (Terre sans pain, Bunuel, 1932) qui peut être reconnu comme un discours marqué par le timbre trop guttural et par sa tonalité étrangement neutre alors même que le commentateur décrit des événements tragiques. Mais l'exemple est en fait extrêmement problématique : dans la version originale espagnole, la voix est troublante pour ceux et celles qui sont attentifs au contraste entre sa sonorité et son apparente froideur; la traduction française évacue quant à elle sa sonorité spécifique pour ne conserver que le ton monocorde, ne laissant guère paraître qu'un commentaire objectivant qui perd de sa spécificité.

Il est pourtant une situation où abondent les voix over marquées par leur timbre trop nasillard et par leur tonalité étrangement claire : on est souvent frappé par la voix qui accompagne les vieux documentaires, que ce soient par exemple des vieux films d'actualités Pathé, des films de reportage telle La Natation par Jean Taris (Vigo, 1931) ou des documentaires à l'exemple de ceux de l'abbé Proulx; pour peu qu'on soit attentif, notre réaction à l'égard de ces films d'autrefois ne tient pas seulement au commentaire qui les accompagne, encore qu'il joue indubitablement un rôle, mais aussi au timbre, au grain de voix et à la tonalité générale de la voix du commentateur. Trop claire pour nos critères actuels, avec des syllabes trop détachées, la matérialité de la voix perce et on écoute d'une oreille critique ou amusée - qui n'est qu'une autre façon de traduire notre position critique - ces discours d'antan.

La tonalité particulière de la voix dans ces films s'explique, notamment, par des caractéristiques techniques : jusqu'au début des années 50, l'enregistrement et le montage sonore s'effectuaient sur 
des pistes optiques, c'est-à-dire que le son était converti sous forme de signaux lumineux et ce depuis la prise de son jusqu'à la copie finale; or le transfert du son en signal optique avait pour effet d'accentuer les hautes fréquences, hautes fréquences qui, par ailleurs, étaient difficiles à rendre en raison des limites inhérentes aux équipements de sonorisation dans les salles; ainsi donc, tout le travail technique décalait le son vers les hautes fréquences; plus encore, on accentuait techniquement ce décalage de façon à améliorer l'intelligibilité des voix. Concrètement, on ajoutait des hautes fréquences pour compenser leur éviction par les appareils de reproduction dans les salles. Ce n'est qu'avec l'avènement progressif du matériel d'enregistrement magnétique (les premiers magnétophones portatifs sont apparus en 1948) et avec l'amélioration des équipements sonores dans les salles que l'enregistrement et le traitement du son acquirent progressivement leur sonorité actuelle 5 . Aujourd'hui, lorsque nous écoutons les films de cette époque, avec des appareils qui sont à même de reproduire les hautes fréquences, nous entendons non seulement le discours de ces commentateurs mais aussi, et peut être surtout, ces voix trop claires, aux intonations quasi chantantes; et frappés par leur matérialité, c'est d'une oreille critique que nous suivons leur discours.

\section{Le positionnement de la voix}

$\mathrm{Si}$ on essaie maintenant de formaliser en termes sémiotiques ce qui survient lorsque, aussi bien au niveau du discours du personnage visualisé qu'au niveau du personnage non visualisé, la voix se démarque par ses caractéristiques matérielles, c'est-à-dire lorsque le timbre et/ou le grain percent et se laissent entendre, on remarque que le trait commun à toutes les situations tient dans le déplacement de notre attention du discours sur son locuteur. Ainsi, on écoute moins le dit que le dire; notre attention est alors portée sur le personnage qui s'exprime et qui devient en quelque sorte le véritable objet d'attention. C'est à ce niveau, me semble-t-il, que se joue la première grande conséquence d'une voix marquée : de sujet, de celui qui a pour fonction de professer un discours, d'agir par sa voix, le locuteur à la voix marquée devient un objet, c'est-àdire quelque chose qu'on observe, qu'on met à distance pour l'examiner.

Au niveau des personnages visualisés, ce déplacement de sujet à objet s'observe aisément : dans les exemples analysés plus haut, on a souligné que tant Mammy, Prissy qu'Amadeus avaient des rôles de second plan au regard des personnages principaux; nul ne s'y trompe, l'héroïne de Gone With the Wind n'est autre que Scarlett O'Hara autour de laquelle gravite Mammy et Prissy, le personnage 
principal d'Amadeus n'est autre que Salieri, Mozart n'étant qu'un objet de jalousie.

Le même phénomène se répète au niveau des personnages non visualisés. Ainsi, le narrateur espagnol de Las Hurdes et les commentateurs des vieux documentaires à l'exemple de celui de $L a$ Natation par Jean Taris cessent d'être, le temps où nous écoutons leur voix pour leurs qualités matérielles, nos guides dans le film : ils deviennent des personnages dont on entend la spécificité sonore.

Ce déplacement de la position sujet vers la position objet a une conséquence directe sur notre perception du discours : le discours s'inscrit normalement dans un contexte dans lequel il est destiné à remplir une fonction, c'est-à-dire que le discours verbal a, à un premier niveau, une valeur illocutoire qui fait que l'on reconnaît qu'il s'agit d'une question, d'un ordre, d'une constatation, etc., mais aussi, à un niveau pragmatique, un effet perlocutoire, c'est-àdire que la question, l'ordre, le constat, etc., sont censés produire un effet chez l'auditeur : ainsi ce dernier répondra, s'exécutera, discutera, etc., suivant l'effet perlocutoire recherché6. Pour prendre un exemple quotidien, si je vous demande l'heure, j'introduis dans mon énoncé un acte illocutoire du type «demander une question», acte illocutoire censé créer, par son effet perlocutoire, un comportement du type «m'indiquer l'heure». Or, comme on l'a souligné à différentes reprises, lorsque le timbre et/ou le grain de la voix sont marqués, il se produit une invalidation du discours, de sorte que la valeur perlocutoire se voit compromise. Dit autrement, le discours se présente comme une réflexion, une interrogation, une réponse, etc., c'est-à-dire qu'on y décèle une valeur illocutoire explicite dans sa texture, mais celle-ci ne produit pas l'effet perlocutoire escompté mais un tout autre effet, celui «d'objectiver» le personnage et son discours. Le discours marqué par le grain et/ou le timbre de la voix devient ainsi un discours dont la portée est compromise, un discours qui ne produit d'autre effet que de faire entendre sa matérialité.

\section{La voix de l'auteur}

Cette analyse est cependant à relativiser par rapport à la narration en voix over de réalisateurs qui inscrivent explicitement leur présence dans la surface textuelle tant leur grain de voix est marqué et reconnu. Pour ne citer que quelques exemples, la voix nasillarde de Guitry dans Si Paris nous était conté (Guitry, 1955), celle de Jean Cocteau dans Le Sang d'un poète (Cocteau, 1931), ou celles de Woody Allen et de François Truffaut qui ponctuent quantité de leurs films, constituent autant de renvois incontournables aux comédiens-réalisateurs pour quiconque est familier avec ces réalisateurs. Pour qualifier cet effet de signature, 
on dira de ces voix qu'elles sont auctoriales. En ce cas, on le voit bien, la caractérisation de la voix a un effet diamétralement opposé au précédent en ce qu'elle attribue au discours une valeur et une portée pour ainsi dire absolues, celles-là même qui sont propres à l'auteur.

\section{Conclusion}

On voit, au terme des ces quelques réflexions, qu'une voix, avant même d'en écouter le discours, influe directement sur notre perception du personnage et de son rôle dans l'économie du film. Et on a vu, tant avec des exemples de personnages visualisés qu'avec des exemples de narrations en voix over, que le timbre de voix nous conduit à adopter une attitude particulière à l'égard du personnage et de son discours, attitude qui place le personnage en position d'objet et non plus de sujet et qui peut, dans certains cas, mettre à mal la dimension perlocutoire du discours : le dire éclipse alors le dit qui devient du coup sans effet. Il est aussi apparu en cours d'analyse que d'autres dimensions telles l'identité sexuelle et l'identité auctoriale influent sur notre perception du locuteur et nous amènent, de façon plus ou moins consciente, à adopter une attitude à l'égard des discours verbaux.

Il m'apparaît ainsi, au terme de ces quelques réflexions, que le choix d'une voix, la détermination d'un casting sonore, contribue à la sonorité du film tout autant que la musique et module non seulement notre perception des personnages et de leurs fonctions, mais aussi notre appréhension de tout le récit. C'est dire qu'il nous faut écouter d'un peu plus près les personnages des films, qu'il est temps que les théoriciens intègrent les éléments spécifiques aux matériaux filmiques dans leurs analyses. C'est au prix de cette attention nouvelle aux qualités des matériaux, d'une nouvelle écoute et d'un nouveau regard que les théoriciens parviendront à dépasser l'application de leurs concepts pour enfin être à l'écoute des films.

\section{Université du Québec à Montréal}

\section{NOTES}

1 L'attribution repose généralement sur le simple synchronisme de la voix avec le mouvement des lèvres du personnage visualisé, voire sur la simple illustration de ce qu'on présente comme étant la source, comme lorsqu'on attribue une voix à un animal ou à une machinc.

2 On a signalé plus avant un quatrième cas de figure possible, soit l'exemple du personnage visualisé dont le visage demeure hors champ. En ce cas, comme dans l'exemple de Kiss me Deadly, ce sont les traits repérables dans la voix et 
plus exactement le grain de la voix qui permettra au spectateur de se construire une image du locuteur.

3 Une exception cependant, qui du reste démarque le film La Lectrice de la production standard : le compagnon de la lectrice intervient en registre over lorsqu'il se reconnaît dans la description d'un personnage du roman qu'elle lit. Dans une narration standard, jamais on n'entend le narrataire en registre over; ici Deville joue avec les conventions en faisant intervenir le compagnon de la lectrice qui dit : "C'est vrai, c'est tout à fait moi», lors de la description du compagnon de la lectrice. Du reste, la correspondance saute pour ainsi dire aux yeux, les deux personnages étant incarnés par le même comédien.

4 La récurrence se vérifie dans les différents "Contes Moraux» : La Boulangère de Monceau (1962), La Carrière de Suzanne (1963), La Collectionneuse (1967), Ma nuit chez Maud (1969), L'Amour l'après-midi (1972). Une exception cependant, le narrateur dans Le Genou de Claire (1970) est réduit à des inscriptions graphiques qui ponctuent le cours des jours tel un journal.

5 Je tiens à remercier Michael Drolet, responsable technique à l'ONF, qui m'a donné toutes ces informations techniques.

6 Pour ces concepts, on verra notamment J. L. Austin, Quand dire c'est faire et Catherine Kerbrat-Orecchioni, L'Implicite.

\section{BIBLIOGRAPHIE}

Austin, John L. Quand dire c'est faire. Paris : Seuil, 1962.

Bailblé, Claude. «Programmation de l'écoute». Cahiers du cinéma 292 (septembre 1978).

Bailblé, Claude. «Programmation de l'écoute 2». Cahiers du cinéma 293 (octobre 1978).

Bailblé, Claude. «Programmation de l'écoute 3». Cahiers du cinéma 297 (février 1979).

Barthes, Roland (1972): «Le Grain de la voix». Musique en jeu 9 (1972). Repris dans L'Obvie et l'obtus. Paris : Seuil, 1982.

Bonitzer, Pascal (1975): «Les Silences de la voix». Cahiers du cinéma 256 (février-mars 1975).

Chion, Michel. La Voix au cinéma. Paris : éd. de l'Étoile/Cahiers du cinéma, 1982.

Chion, Michel. Le Son au cinéma. Paris : éd. de l'Étoile/Cahiers du cinéma, 1985.

Chion, Michel. La Toile trouée. Paris : éd. de l'Étoile/Cahiers du cinéma, 1988.

Chion, Michel. L'Audio-vision. Paris : Nathan, 1990.

Doane, Mary Ann. «The Voice in the Cinema: The Articulation of Body and Space». Yale French Studies 60 (1980).

Gaudreault, André et François Jost. Cinéma et Récit II. Le Récit cinématographique. Paris : Nathan, 1990.

Kerbrat-Orecchioni, Catherine. L'Implicite. Paris : Armand Colin, 1986.

Kozloff, Sarah. Invisible Storytellers. Voice-over Narration in American Fiction Film. Berkeley : University of California Press, 1988. 